\title{
Laser Resection of Subglottic Pleomorphic Adenoma
}

\author{
${ }^{1} \mathrm{D}$ Nair, ${ }^{2} \mathrm{P}$ Pai, ${ }^{2} \mathrm{P}$ Chaturvedi, ${ }^{3} \mathrm{R}$ Kumar \\ ${ }^{1}$ Assistant Professor, Department of Head and Neck Surgery, Tata Memorial Hospital, Mumbai, Maharashtra, India \\ ${ }^{2}$ Associate Professor, Department of Head and Neck Surgery, Tata Memorial Hospital, Mumbai, Maharashtra, India \\ ${ }^{3}$ Department of Head and Neck Surgery, Tata Memorial Hospital, Mumbai, Maharashtra, India
}

Correspondence: Deepa Nair, Assistant Professor, Department of Head and Neck Surgery, Tata Memorial Hospital, Mumbai Maharashtra, India, e-mail: drdeepanair@hotmail.com

\begin{abstract}
Tumors arising from the minor salivary glands in the larynx are rare. The most common tumors occurring here are adenoid cystic carcinomas. ${ }^{1}$ Among the benign tumors, pleomorphic adenoma is the most common. ${ }^{1}$ We present a case of subglottic pleomorphic adenoma which was managed by endolaryngeal endoscopic $\mathrm{CO}_{2}$ laser surgery. Laser as a primary treatment modality for the treatment of subglottic pleomorphic adenomas has not been well-described, as only one case has been reported in literature using this modality.
\end{abstract}

Keywords: Subglottic, Salivary gland tumor, Pleomorphic adenoma, Laser surgery.

\section{CASE PRESENTATION}

A 48 years old male was referred to our center, a specialized tertiary comprehensive cancer center with a history of progressively worsening dyspnea since 6 months. The patient was admitted in a general hospital for stridor and treated with steroids following which his stridor subsided. Further investigations with rigid laryngoscopy under general anesthesia revealed a subglottic mass. The biopsy was reported as pleomorphic adenoma and he was referred to us for further management.

On presentation, the patient had no stridor or hoarseness. On flexible laryngoscopy, a pedunculated mass with a broad base was seen arising $1 \mathrm{~cm}$ below the level of the vocal cords, extending inferiorly for about $1 \mathrm{~cm}$ and occluding 30\% of the subglottic lumen. Both the vocal cords were normal in appearance and mobility (Fig. 1). The rest of the tracheobronchial tree was normal. A computed tomography (CT) scan of the larynx revealed a lobulated mass in the subglottis arising from the posterior wall measuring $1.2 \times$ $1.2 \times 1.4 \mathrm{~cm}$, extending anterior and abutting the right lateral wall of subglottis with no erosion of the underlying cartilage (Figs 2 and 3). The patient underwent endolaryngeal endoscopic $\mathrm{CO}_{2}$ laser (Lumenis ${ }^{\mathrm{TM}}$ Israel, $3.5 \mathrm{~W}$, superpulse mode) resection of the mass under general anesthesia using apnea technique. The base of the mass was completely resected with wide margins. The cricoid cartilage was not exposed. Postoperative recovery was uneventful.

The histopathology report demonstrated a biphasic tumor in submucosa consisting of epithelial nests, trabeculae and tubules embedded in a fibromyxoid stroma (Fig. 4A). The epithelial component composed of 2 cell types: epithelial cells arranged in duct like structures or cords and myoepithelial cells situated at around the ducts and in solid

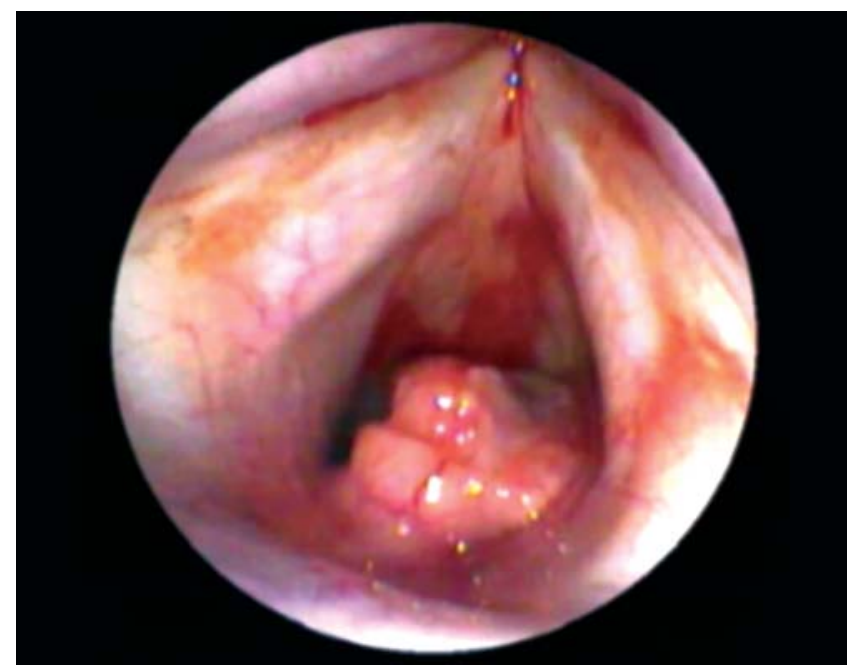

Fig. 1: Flexible laryngoscopy picture showing presence of pedunculated mass arising from posterior wall of subglottis, compromising laryngeal lumen 


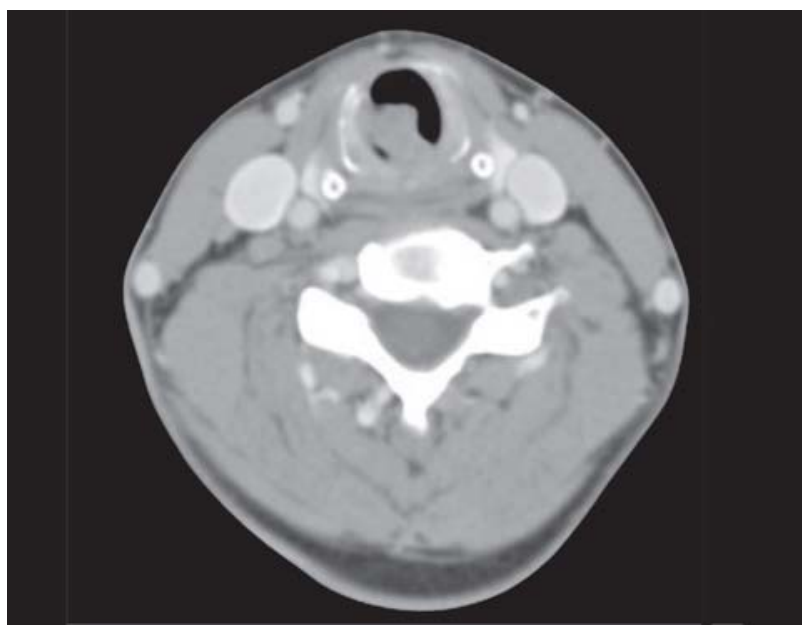

Fig. 2: Axial computed tomography (CT) image showing presence of mass in posterior wall of subglottis with no cartilage erosion

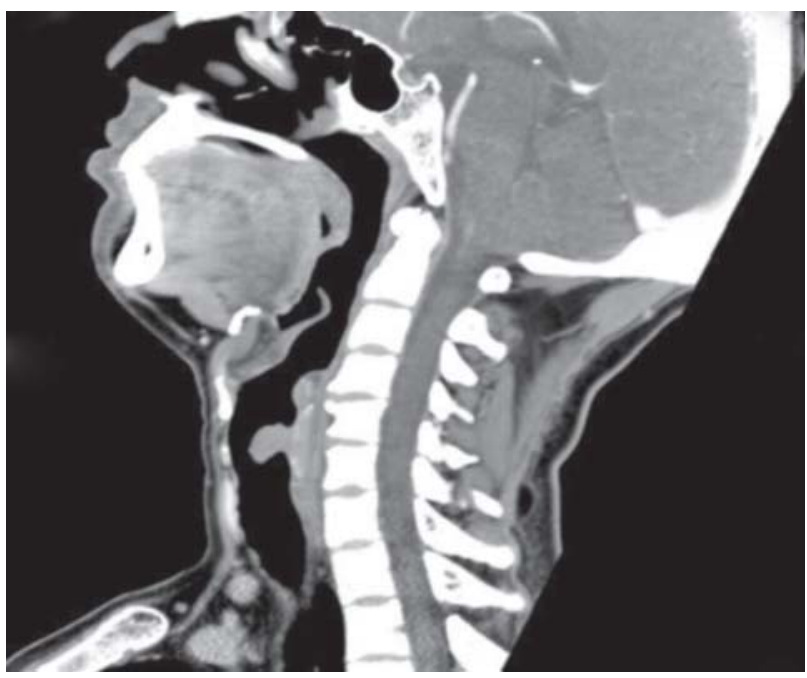

Fig. 3: Sagittal computed tomography (CT) image showing mass arising from posterior wall of subglottis

nests. Focal stromal hyalinization was noted adjacent to myoepithelial cells. The relative proportion of the epithelial and stromal components was variable, with focal increase in cellularity at places. At higher magnification, the epithelial cells appeared uniform with minimal cytologic atypia (Fig. 4B), thus suggestive of pleomorphic adenoma of minor salivary gland. On immunohistochemistry, CK, p63 and Calponin stained positive for the tumor confirming the diagnosis.

Patient is asymptomatic, free of disease with a normal voice 14 months on follow-up.

\section{DISCUSSION}

In the larynx, minor salivary gland tumors rarely occur and constitute less than $1 \%$ of laryngeal tumors. They arise from
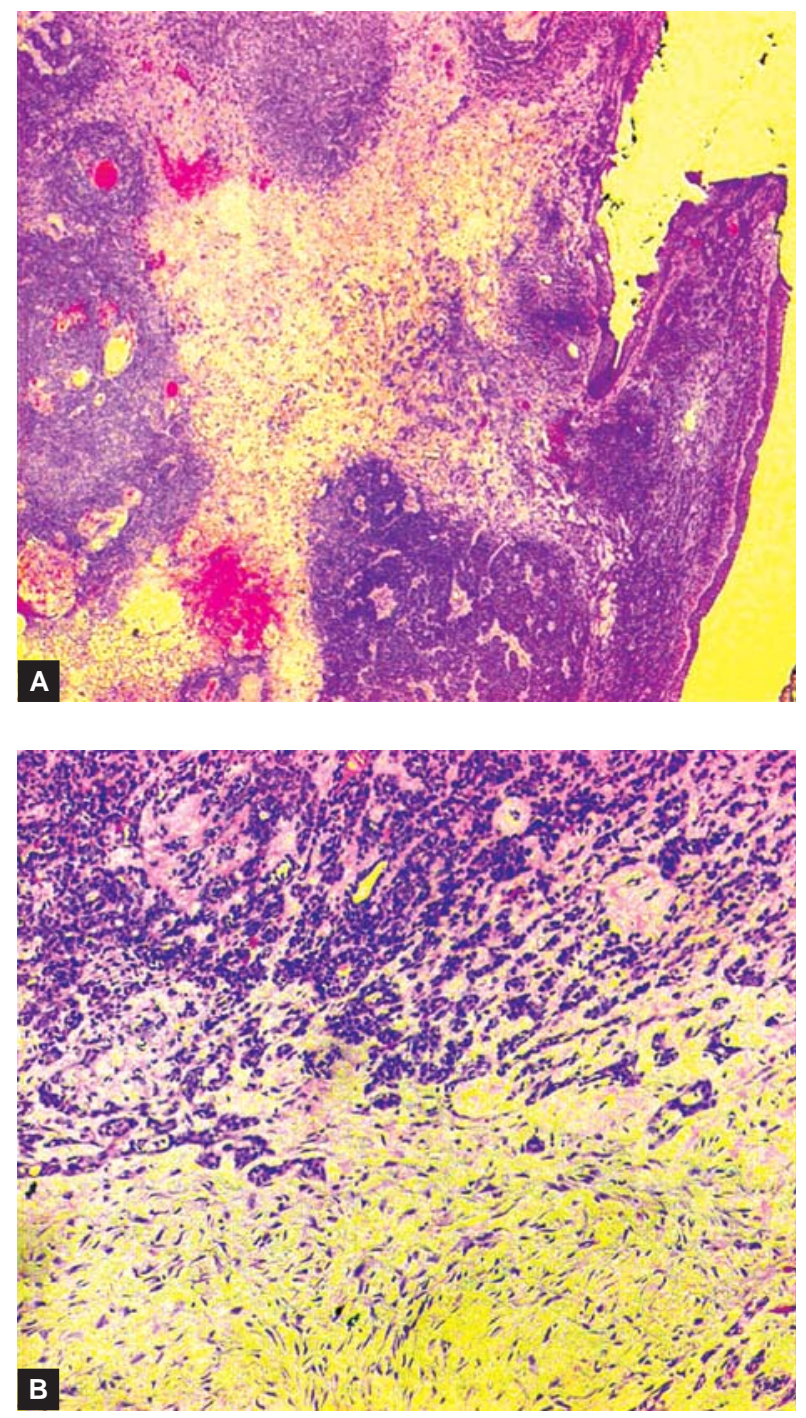

Figs $4 \mathrm{~A}$ and $\mathrm{B}$ : $\mathrm{A}$ biphasic tumor that is composed of bland epithelial cells intricately admixed with a fibromyxoid stroma (Fig. 4A; H\&E 40X, Fig. 4B; H\&E 100X)

submucosal glands, and in the larynx there is a distinct anatomical distribution. ${ }^{2}$ The largest numbers are in the subglottis; however, they also occur in the supraglottis in the false cords, aryepiglottic folds, and caudal aspect of the epiglottis. In the glottis, they are most commonly located in the floor of the sinus of Morgagni and subglottic surface of the anterior commissure. Among the various salivary gland tumors of the larynx, the most common tumors occurring are adenoid cystic carcinomas. ${ }^{1}$ Among the benign tumors, pleomorphic adenoma is the most common.

Most tumors commonly present as submucosal subglottic masses of which around 20 cases have been reported in literature. ${ }^{3-6}$ 
These tumors are more commonly seen the third to sixth decades of life, as was our patient. ${ }^{2}$ Tumors of the supraglottis present with dysphagia and hoarseness, whereas airway compromise is more frequently associated with subglottic tumors. ${ }^{6}$ Our patient initially presented with dyspnea and stridor which reduced following biopsy performed at another center. As there was no involvement of the true or false cords, the patient always had a normal voice. Any stridorous patient with normal cord mobility not responding to bronchodilators must be evaluated by flexible laryngobronchoscopic examination for presence of a mass obstructing the subglottic or tracheal airway.

Histopathological examination revealed the mass to be pleomporphic adenoma which consists of myxoid stroma with acini, cords as well as a ratio of epithelial and myoepithelial cells ${ }^{6}$. However, sometimes it is difficult to interpret small biopsies and differentiate benign from malignant lesions especially carcinoma ex pleomorphic adenoma. The differential diagnosis of tumors in this area include carcinomas, hemangiomas, cylindromas, fibromas, chondromas and paragangliomas. ${ }^{7}$

The treatment of these lesions is wide excision. A variety of approaches have been described in literature. Pharyngotomy ${ }^{3,4}$ has been advocated especially for supraglottic lesions. Laryngofissure ${ }^{5}$ has been used in patients with glottic or subglottic disease. Only one reference to the use of $\mathrm{CO}_{2}$ laser for excision of subglottic mass ${ }^{8}$ has been found in literature. In our case, we could adequately expose the disease by suspending the larynx and hence opted for endolaryngeal laser resection. We were able to completely excise the tumor with adequate margins. After removing the bulky part of the tumor, its broad base was separately excised to ensure the completeness of excision. The underlying cartilage was free requiring no further excision.

Usage of $\mathrm{CO}_{2}$ laser for the resection ensures complete resection of tumor with minimal thermal damage to the vocal cords or arytenoids, thereby preventing any post-treatment sequelae. Our patient had no deterioration in voice or swallow after the surgery.

In all the reported cases, no recurrences have been found. However, due to the theoretical possibility of local recurrence and malignant change, it is better to keep the patient on close observation and life long follow-up.

\section{CONCLUSION}

Subglottic pleomorphic adenomas are rare causes for fixed respiratory obstruction. This diagnosis must be kept in mind in the cases of subglottic masses and proven by surgery. Wide excision of the lesion with safe margins with minimal damage to surrounding structures is feasible with endolaryngeal endoscopic surgery.

\section{REFERENCES}

1. Bastakis JG. Tumors of the head and neck. Clinical and pathological considerations (2nd ed). Baltimore: Williams and Wilkins, 1979;8-9.

2. Bak-Pedersen K, Nielson KO. Subepithelial mucous glands in the adult human larynx: Studies on number, distribution and density. Acta Otolaryngol 1986;102:341-52.

3. Som PM, Nagel BD, Fererstein SS, et al. Benign pleomorphic adenoma of the larynx. A case report. Ann Otol Rhinol Laryngol 1979;27:714-18.

4. Dubey SP, Banerjee S, Ghosh LM, et al. Benign pleomorphic adenoma of the larynx: Report of a case and review and analysis of 20 additional cases in the literature. ENT- Ear, Nose and Throat Journal 1997;76(8):548-57.

5. Sawatsubashi M, Tuda K, Tokunaga O, Shin T. Pleomorphic adenoma of the larynx: A case and review of the literature in Japan. Otolaryngology_Head and Neck Surgery 1997; 117(4):415-17.

6. Zakzouk MS. Pleomorphic adenoma of the larynx. J Laryngol Otol 1985;99:611-16.

7. Maisel R, Schmidt D, Pambuccian S. Subglottic laryngeal paraganglioma. Laryngoscope 2003;113(3):401-05.

8. Berjis N, Haji Malian M. Pleomorphic adenoma presented as a subglottic mass. Shiraz E-Medical Journal. July 2006;7(3). 